\title{
Atenção primária à saúde em tempos de COVID-19: o que fazer?
}

\author{
Primary healthcare in times of COVID-19: \\ what to do?
}

\author{
Atención primaria en salud en tiempos de la \\ COVID-19: ¿qué debemos hacer?
}

\author{
Maria Guadalupe Medina 1 \\ Lígia Giovanella 2 \\ Aylene Bousquat 3 \\ Maria Helena Magalhães de Mendonça 2 \\ Rosana Aquino 1 \\ Comitê Gestor da Rede de Pesquisa em Atenção \\ Primária à Saúde da Abrasco 4
}

doi: 10.1590/0102-311X00149720
A pandemia de COVID-19 é um desafio sem precedentes para a ciência e para a sociedade, cobrando respostas rápidas e diversas dos sistemas de saúde que precisam ser reorganizados, em todos os seus componentes, para o seu enfrentamento.

No Brasil, e em diversos países do mundo, a resposta sanitária tem sido centrada nos serviços hospitalares, com ações para a ampliação do número de leitos, especialmente, de unidades de tratamento intensivo e respiradores pulmonares. Sem retirar a importância da adequada estruturação da atenção especializada voltada aos casos mais graves da COVID-19, é preciso alertar que, no âmbito da atenção primária à saúde (APS), muito pode e precisa ser feito.

$\mathrm{Na}$ ausência de vacinas e de medicamentos específicos e devido à alta transmissibilidade da infecção, as únicas intervenções eficazes para o controle da pandemia são medidas de saúde pública como isolamento, distanciamento social e vigilância dos casos, com o propósito de reduzir o contágio, evitando sofrimento e morte, ao frear a velocidade da pandemia. Ao mesmo tempo é necessário dotar o sistema de recursos para oferecer a atenção adequada e oportuna 1.

Nesse sentido, a reorganização dos serviços de APS para, simultaneamente, enfrentar a epidemia e manter a oferta regular de suas ações é imperativa, e seu necessário protagonismo e readequação vêm sendo destacados em documentos e relatórios produzidos no país 2,3. Mesmo reconhecendo as diversas fragilidades de atuação das equipes, ressalta-se que a Estratégia Saúde da Família (ESF) é o modelo mais adequado por seus atributos de responsabilidade territorial e orientação comunitária, para apoiar as populações em situação de isolamento social pois, mais do que nunca, é preciso manter o contato e o vínculo das pessoas com os profissionais, responsáveis pelo cuidado à saúde.

Este artigo analisa possibilidades de atuação dos serviços de APS na rede do Sistema Único de Saúde (SUS) que contribuam para o controle da epidemia e, simultaneamente, cumpram com a sua função essencial de garantir atenção cotidiana e capilarizada.

\section{Eixos de intervenção da APS}

O enfrentamento à pandemia exige a elaboração de planos de gerenciamento de risco 4 em vários níveis (nacional, estadual, municipal e local), fortalecendo a atuação no território, que considere: a população a ser acompanhada (casos leves de COVID-19 e outros problemas de saúde); a adequada proteção
1 Instituto de Saúde Coletiva, Universidade Federal da Bahia, Salvador, Brasil. 2 Escola Nacional de Saúde Pública Sergio Arouca, Fundação Oswaldo Cruz, Rio de Janeiro, Brasil.

${ }^{3}$ Faculdade de Saúde Publica, Universidade de São Paulo, São Paulo, Brasil.

4 Outros membros listados ao final do artigo.

Correspondência M. G. Medina Instituto de Saúde Coletiva, Universidade Federal da Bahia.

Rua Basílio da Gama s/n, Salvador, BA 40110-040, Brasil.

medina@ufba.br 
dos profissionais de saúde, com condição segura à realização do seu trabalho, evitando, também, que sirvam de fonte de contaminação; as mudanças organizacionais compatíveis com a realidade local; as necessidades de apoio logístico e operacional (incluindo transporte, material e equipamentos de segurança e proteção); formação e educação permanente dos profissionais de saúde; mapeamento de potencialidades e dificuldades de cada território; a retaguarda necessária a uma ação coordenada da APS com outras instituições e serviços de saúde no território de abrangência das equipes ou fora dele; e parcerias com as organizações comunitárias, potencializando habilidades e estimulando a solidariedade.

A atuação da APS pode ser sistematizada em quatro eixos: (i) vigilância em saúde nos territórios; (ii) atenção aos usuários com COVID-19; (iii) suporte social a grupos vulneráveis; e (iv) continuidade das ações próprias da APS.

\section{Vigilância em saúde nos territórios}

Visando a bloquear e reduzir o risco de expansão, a APS deve estar envolvida no gerenciamento de risco da epidemia, atuando de forma articulada com a vigilância em saúde dos municípios, estabelecendo fluxos de informação, em uma via de mão dupla, para aprimorar a qualidade das ações.

A notificação, detecção e acompanhamento dos casos, com isolamento domiciliar dos casos e quarentena dos contatos são atividades centrais de mitigação da epidemia, a serem desenvolvidas pelas equipes de APS.

O isolamento social pode ser incentivado por todos os profissionais da equipe, principalmente pelos agentes comunitários de saúde (ACS), mobilizando lideranças e recursos locais com ampla divulgação de informações e realização de medidas concretas. A literatura tem mostrado que os ACS são importantes aliados no enfrentamento de epidemias 5, especialmente no que diz respeito à conscientização da população e combate ao estigma relacionado à doença, o que realça o seu papel na difusão de informações corretas sobre a prevenção de COVID-19, no combate às fake news e no apoio a atividades educativas no território, relacionadas à higiene e proteção de trabalhadores e usuários nos diversos equipamentos sociais, de modo que se constituam em ambientes seguros para a população.

\section{Atenção aos usuários com COVID-19}

Tem sido implementada a organização de fluxos distintos para o cuidado dos pacientes com quadros leves, separando os sintomáticos respiratórios dos usuários com outros problemas que necessitam de cuidado presencial, identificando e orientando indivíduos com maior risco de desenvolver quadros graves e garantindo o encaminhamento oportuno daqueles que necessitam de cuidados de outros níveis de atenção 6,7.

Ademais, as modalidades de atendimento on-line têm sido priorizadas e bastante difundidas, o que impõe a necessidade de se estender o acesso à telefonia celular e Internet de forma rápida para profissionais e usuários. A consulta remota deve ser realizada com base em protocolos, com mensagens claras e objetivas, dando preferência ao vídeo, pois facilita o monitoramento de sinais vitais 8 .

A qualidade do atendimento na APS e a continuidade do cuidado aos pacientes com COVID-19 só podem ser asseguradas com recursos adequados que garantam a segurança do paciente e resolubilidade do problema. Na avaliação da gravidade dos casos e de sua evolução, a medição do nível de saturação de oxigênio no sangue é importante, sendo necessário disponibilizar oxímetro para as equipes, para uso na triagem, atendimento presencial e em domicílio, no acompanhamento de casos suspeitos e confirmados. Quanto à continuidade do cuidado ao paciente, é mister que serviços de APS estejam integrados à rede de emergência, hospitalar e de transporte sanitário, associada à regulação de leitos com definição de fluxos e canais de comunicação abertos e ágeis, para a garantia de cuidado oportuno, conforme a gravidade.

\section{Suporte social a grupos vulneráveis}

A ESF tem ampliado sua atuação na resposta às necessidades de populações socialmente vulneráveis e de grupos de risco, como idosos e indivíduos que apresentam comorbidades, que vivem cotidianamente situações de isolamento ou restrições, agora agravadas. 
Para que possa, efetivamente, seguir as recomendações preventivas relacionadas à COVID-19, essa população necessitará de todo tipo de apoio (sanitário, financeiro, psicológico e social), incluindo atendimento pela rede de serviços de saúde e acesso aos mecanismos de proteção social.

Encontrar as melhores soluções para os problemas graves e diversos dos grupos populacionais mais vulneráveis exige uma ação coordenada no território com as lideranças, equipamentos e instituições locais, articulando as ações implementadas pelas equipes com as iniciativas comunitárias, muitas já em andamento, destacando-se o engajamento comunitário como uma importante estratégia global no enfrentamento da pandemia 9. Podem ser destacadas propostas como a oferta de abrigo em hotéis, escolas ou outros equipamentos para pessoas em risco (idosos, doentes crônicos solitários, mulheres vítimas de violência e população de rua) e apoio domiciliar para idosos que terão dificuldades de se manter e de se cuidar. Iniciativas de engajamento comunitário vêm sendo observadas em experiências de solidariedade, em que as próprias comunidades estão promovendo mutirões de distribuição de cestas básicas, confecção de máscaras por artesãs locais e uso de escolas para o isolamento de casos leves de COVID-19 10.

\section{Continuidade das ações próprias da APS}

As atividades de rotina da APS precisam ser preservadas em tempos de pandemia, até porque as previsões apontam para um longo curso de convivência com o novo vírus, com alternância de maior e menor isolamento social, o que exige readequação de certos procedimentos e incorporação de outros para que a APS funcione cumprindo sua missão, incluindo novas formas de cuidado cotidiano à distância, evitando o risco de aprofundamento da exclusão do acesso e das desigualdades sociais.

O uso de tecnologias de informação e comunicação, como WhatsApp e telefone, para a realização de teleconsulta, garante a oferta de ações de forma segura, de modo que não haja descontinuidade e agravamento das condições dos usuários em tratamento. Sugere-se responder a demandas frequentes de usuários - como a renovação de receitas e a busca por medicamentos - de modo que estes não precisem se dirigir à unidade básica de saúde (UBS), seja prolongando o tempo de duração das prescrições, seja viabilizando a entrega domiciliar dos medicamentos pelo ACS, adotando-se os cuidados necessários.

Buscar contatar por telefone os pacientes pré-agendados e realizar teleconsulta com médicos, enfermeiros da equipe ou médicos residentes e internos, e a criação de agenda para teleconsulta são iniciativas sugeridas por experiências em curso 11 , lembrando-se que, para alguns, serão mantidos atendimentos presenciais, assim como outras atividades de rotina, a exemplo da vacinação que precisa ser realizada sem expor a população ao risco de contaminação.

\section{Considerações finais}

O fracasso de experiências internacionais de tentativas de enfrentamento da pandemia centradas no cuidado individual hospitalar alertou para a necessidade de uma abordagem mais territorializada, comunitária e domiciliar, e a necessidade de ativar a APS, forte e integral, em toda a sua potencialidade. O modelo brasileiro, com suas equipes de saúde da família e enfoque territorial, apresentou impactos positivos na saúde da população e tem papel importante na rede assistencial de cuidados, além de poder contribuir vigorosamente para a abordagem comunitária, necessária no enfrentamento de qualquer epidemia.

Não obstante essa potencialidade, as tentativas de desmantelamento da ESF, desde 2017, com redução de agentes comunitários de saúde, flexibilização de carga horária de profissionais, abolição da prioridade para a ESF, extinção dos Núcleo Ampliado de Saúde da Família e Atenção Básica (NASF-AB), perda de profissionais com a finalização do Programa Mais Médicos, desincentivos a abordagem territorial com o novo modelo de financiamento da atenção básica com base em número de cadastrados, fragilizando o enfoque comunitário, entre outros ${ }^{12}$, representam constrangimentos importantes para uma atuação adequada da APS no enfrentamento da pandemia.

Essas ameaças recentes foram somadas a problemas cronicamente enfrentados na APS brasileira. A precariedade das relações trabalhistas em grande parte das equipes, a prática de contratação de 
organizações sociais e de outras formas de privatização da gestão de unidades básicas de saúde que mercantiliza as relações e fragiliza vínculos, problemas organizacionais com baixa integração entre a APS e outros níveis de atenção na rede regionalizada comprometendo a coordenação e continuidade com a fragmentação do cuidado, a insuficiente mediação de ações intersetoriais para incidir na determinação social, promover a saúde e reduzir as desigualdades, são alguns dos obstáculos para a exigida atuação da ESF neste momento 13 .

A crise sanitária atual amplificou as debilidades existentes e tem requerido recursos extraordinários da União para os estados e municípios, ainda insuficientes para apoiar as ações de vigilância e cuidado da população. Com todos esses obstáculos, a presença de mais de 40 mil equipes de ESF em todo país, ainda que por vezes incompletas, 260 mil ACS, 26 mil equipes de saúde bucal, cerca de 5 mil NASF, representam as bases do SUS e devem ser fortalecidas se almejamos obter êxito no enfrentamento desta pandemia.

Com todas as dificuldades, é preciso reconhecer que a capilaridade e pujança da força de trabalho da ESF, além das inúmeras bem-sucedidas experiências municipais e locais, têm mostrado a força e resiliência das equipes de SF nos mais diversos contextos.

Mais do que nunca precisamos de uma APS no SUS forte, vigilante, capilarizada, adaptada ao contexto e fiel a seus princípios. A atual crise global é sanitária, política, econômica e social, e exige inovação nos modos de operação e radicalização da lógica de intervenção comunitária no exercício de novas formas de sociabilidade e de solidariedade.

\section{Colaboradores}

Todos os autores participaram da concepção e redação do artigo.

\section{Informações adicionais}

ORCID: Maria Guadalupe Medina (0000-00017283-2947); Lígia Giovanella (0000-0002-6522545X); Aylene Bousquat (0000-0003-2701-1570); Maria Helena Magalhães de Mendonça (00000002-3917-9103); Rosana Aquino (0000-00033906-5170).

\section{Outros membros do Comitê Gestor da Rede de Pesquisa em Atenção Primária à Saúde da Abrasco}

Carlos Leonardo Cunha, Claunara S. Mendonça, Cristiane Spadacio, Daniela L. Carcereri, Elaine Thumé, Elaine Tomasi, Fúlvio Borges Nedel, Geraldo Cunha Cury, Luiz Augusto Fachinni, Márcia Guimarães de Mello Alves, Maria Catarina Salvador da Motta, Sandro Schreiber de Oliveira, Sonia Acioli.

\section{Referências}

1. Aquino E, Silveira IH, Pescarini J, Aquino R, Souza-Filho JA. Medidas de distanciamento social no controle da pandemia de COVID-19: potenciais impactos e desafios no Brasil. Ciênc Saúde Colet 2020; 25 Suppl 1:2423-46.

2. Rede de Pesquisa em APS da Abrasco. Desafios da APS no SUS no enfrentamento da Covid-19. Relatório. In: Seminário Virtual Rede APS/ABRASCO. https://redeaps.org.br/ wp-content/uploads/2020/04/Relatorio-Re de-APS-_Semina\%CC\%81rio-APS-no-SUS-eCovid-16-Abril-2020-final.pdf (acessado em 12/Jun/2020).

3. Engstrom E, Giovanella L, Melo E, Mendes A, Grabois V, Mendonça MHM. Recomendações para a organização da atenção primária à saúde no SUS no enfrentamento da COVID-19. https://portal.fiocruz.br/documento/recomen dacoes-para-organizacao-da-aps-no-sus-no -enfrentamento-da-covid-19 (acessado em 12/ Jun//2020).

4. Dunlop C, Howe A, Li D, Allen LN. The coronavirus outbreak: the central role of primary care in emergency preparedness and response. BJGP Open 2020; 4:bjgpopen20X101041.

5. Bhaumik S, Moola S, Tyagi J, Nambiar D, Kakoti M. Community health workers for pandemic response: a rapid evidence synthesis. medRxiv 2020; 2 mai. https://www.medrxiv.org/conten t/10.1101/2020.04.28.20082586v1. 
6. Guimarães FG, Carvalho TML, Bernardes RM, Pinto JM. A organização da atenção primária à saúde de Belo Horizonte no enfrentamento da pandemia COVID-19: relato de experiência. APS em Revista 2020; 2:74-82.

7. Chan PF, Lai KPL, Chao DVK, Fung SCK. Enhancing the triage and cohort of patients in public primary care clinics in response to the coronavirus disease 2019 (COVID-19) in Hong Kong: an experience from a hospital cluster. BJGP Open 2020; 4:bjgpopen20X101073.

8. Greenhalgh T, Koh GCH, Car J. Covid-19: a remote assessment in primary care. BMJ 2020; 368:m1182.

9. World Health Organization; United Nations Children's Fund. Community-based health care, including outreach and campaigns, in the context of the COVID-19 pandemic. Interim guidance. https://www.who.int/publicationsdetail/community-based-health-care-includ ing-outreach-and-campaigns-in-the-contextof-the-covid-19-pandemic (acessado em 28/ Mai/2020)
10. Melo ML. Sem ação do governo, Alemão cria gabinete de crise com funk contra corona. UOL Notícias 2020; 21 mar. https://noticias.uol.com. $\mathrm{br} /$ cotidiano/ultimas-noticias/2020/03/21/co ronavirus-alemao-cria-gabinete-de-crise-comfunk-da-prevencao-e-doacoes.htm (acessado em 28/Mai/2020).

11. Mendonça CS, Rosset I, Gonçalves MR, Bastos CGM, Medeiros AF. Resposta assistencial de um serviço docente assistencial de APS à pandemia do COVID-19. APS em Revista 2020; 2:28-32.

12. Giovanella L, Franco CM, Almeida PF. Política Nacional de Atenção Básica: para onde vamos? Ciênc Saúde Colet 2020; 25:1475-82.

13. Rede de Pesquisa em Atenção Primária à Saúde da Abrasco. Contribuição para uma agenda política estratégica para a atenção primária à saúde no SUS. Saúde Debate 2018; 42(n.spe 1):406-30. 